\title{
PAPER
}

\section{Effects of room shape and diffusing treatment on the measurement of sound absorption coefficient in a reverberation room}

\author{
Emi Toyoda $^{1, *}$, Shinichi Sakamoto ${ }^{2}$ and Hideki Tachibana ${ }^{2}$ \\ ${ }^{1}$ Kobayasi Institute of Physical Research, \\ 3-20-41, Higashi-motomachi, Kokubunji, 185-0022 Japan \\ ${ }^{2}$ Institute of Industrial Science, University of Tokyo, \\ 4-6-1, Komaba, Meguro-ku, Tokyo, 153-8505 Japan
}

(Received 28 August 2003, Accepted for publication 12 December 2003 )

\begin{abstract}
In this paper, the measurement of sound absorption coefficient of acoustical materials in a reverberation room is investigated by numerical analysis based on the ray-tracing method. As a result, it has been confirmed that the sound absorption coefficient of the same specimen can much differ by the differences of room shape and by the effect of the sound diffusers. In order to evaluate the degree of sound diffusivity in a reverberation room, the angular dependence of the sound power incident to the room boundary and to the surface of the specimen have been calculated. From the result of the numerical analysis, the relationship between the measurement of sound absorption coefficient of materials and the diffusivity of the sound field in a reverberation room has been examined.
\end{abstract}

Keywords: Room shape, Diffusing treatment, Sound absorption coefficient in a reverberation room, Diffusivity, Ray-tracing method

PACS number: 43.55.Br, 43.55.Ka, 43.55.Nd [DOI: 10.1250/ast.25.255]

\section{INTRODUCTION}

The measurement of sound absorption coefficient of acoustical materials in a reverberation room is based on the ideal assumption of diffuse sound field. In reality, however, the degree of sound diffusivity in a reverberation room is highly dependent on the room shape and the arrangement of diffusion elements in the room. Thus the results of the sound absorption coefficient of the same material measured in different reverberation rooms can much differ [1-3].

As a method to realize a highly diffuse sound field, reverberation rooms with irregular shape without any parallel walls are often used [4]. As another method to achieve diffuse sound field, various types of sound diffusers are also used as is recommended in ISO 354 [5] and JIS A 1409 [6].

Although a lot of investigations have been performed so far regarding the sound diffusion in a reverberation room and the method to realize diffuse sound field, the relationship between the measurement accuracy/reproducibility of sound absorption coefficient of materials and the sound field in a reverberation room is still remaining as an important problem. In this paper, therefore, these points are investigated by numerical analysis based on the ray-tracing method.

\section{THEORY}

\subsection{Sound Energy in a Closed Sound Field}

Let us suppose a closed sound field as shown in Fig. 1 which contains an omni-directional sound source. The sound absorption coefficient of each room boundary $\left(w_{1}, w_{2}, \cdots, w_{L}\right)$ is $\alpha_{1}, \alpha_{2}, \cdots, \alpha_{L}$, respectively. Here, it is assumed that the sound source radiates $M$ sound particles in equal angular spacing at the starting time $t=0$. Each particle has equal unit energy (1) and reflects specularly on the room boundaries. When a particle reflects on the boundary $w_{i}$, it loses energy in proportion to the sound absorption coefficient $\alpha_{i}$ of the boundary. Under such assumptions, the position of each particle is calculated by the ray tracing method at a time interval of $\Delta t$.

The energy of the sound particle incident to the boundary $w_{i}$ with the incidence angle between $(j-1) \Delta \theta$ and $j \Delta \theta$ during the time interval between $(n-1) \Delta t$ and $n \Delta t$ is denoted as $\Delta E_{i}(j \Delta \theta, n \Delta t)$. Then, the absorbed energy on the boundary $w_{i}$ during the time interval is expressed as follows.

*e-mail: toyoda@kobayasi-riken.or.jp 


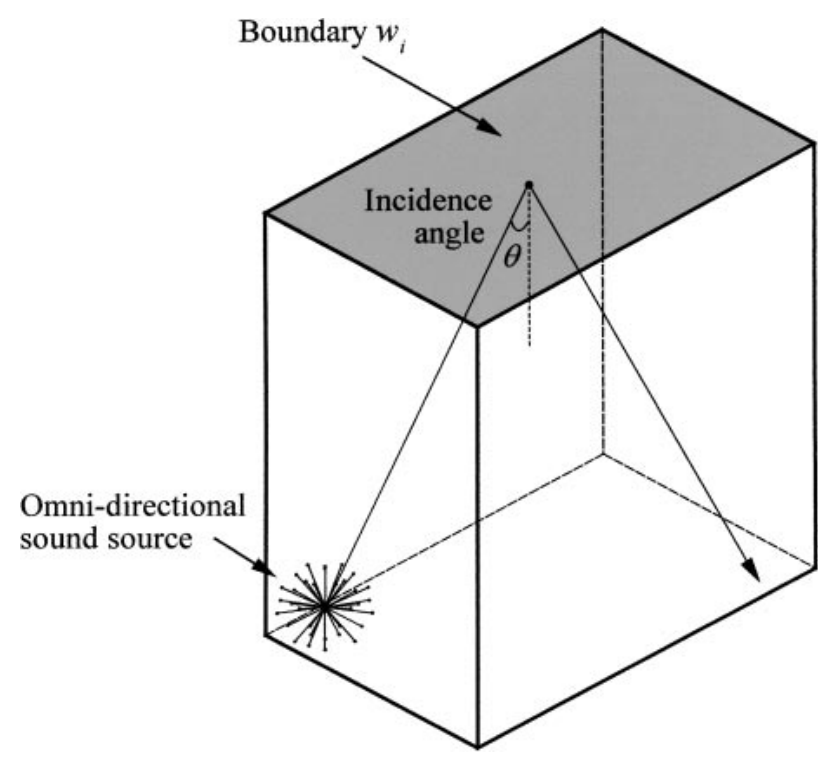

Fig. 1 Closed sound field for calculation.

$$
A_{i}(n \Delta t)=\alpha_{i} \sum_{j} \Delta E_{i}(j \Delta \theta, n \Delta t)
$$

Here, the total energy contained in the room at the time $n \Delta t$ is the difference between the total energy $M$ emitted from the sound source and the energy absorbed on the room boundaries until the time $n \Delta t$, as follows.

$$
E(n \Delta t)=M-\sum_{k=1}^{n} \sum_{i=1}^{L} A_{i}(k \Delta t)
$$

This value indicates the time transition of the total energy contained in the room.

Next, let us consider the sound field in which the sound source radiates a continuous sound. In the discrete time system under consideration here, the sound source radiating a continuous sound can be treated as a source radiating energy pulses intermittently at a time interval of $\Delta t$ as shown in Fig. 2. For such a source condition, the total sound energy existing in the room at the time $n \Delta t$ is expressed as follows.

$$
E_{\mathrm{g}}(n \Delta t) \sum_{k=0}^{n} E(k \Delta t)
$$

This value indicates the growth of the sound energy in the room. As $n$ approaches infinity, the value $E_{\mathrm{g}}(n \Delta t)$ approaches the sound energy under steady state condition $E_{\mathrm{s}}$ as follows.

$$
E_{\mathrm{s}}=\lim _{n \rightarrow \infty} E_{\mathrm{g}}(n \Delta t)
$$

When the source stops radiating the energy pulse at the time $N \Delta t$, the time transition of the sound energy remaining in the room is expressed as follows.

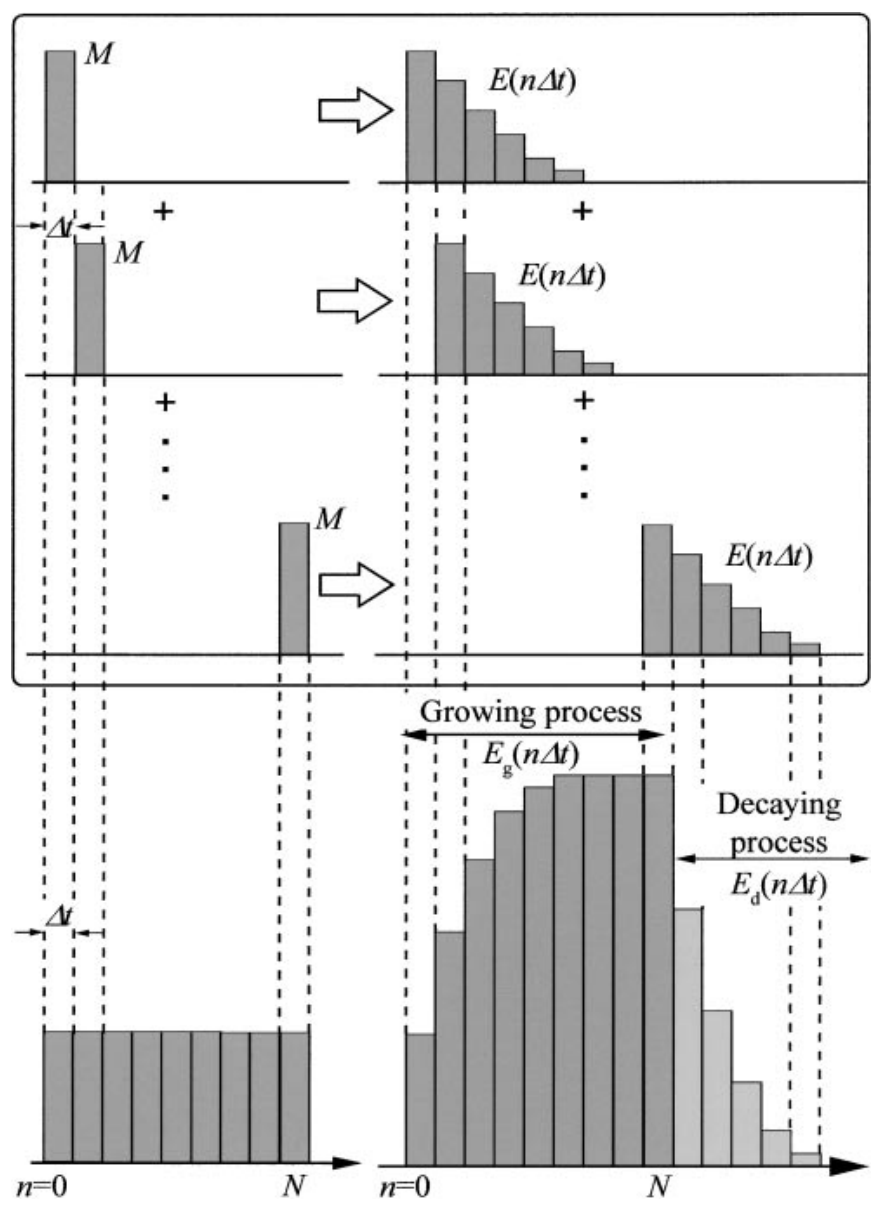

Fig. 2 Growing process $E_{\mathrm{g}}(n \Delta t)$ and decaying process $E_{\mathrm{d}}(n \Delta t)$ under the condition that the sound source radiates energy pulses intermittently at a time interval of $\Delta t$ and stops radiating at the time $N \Delta t$.

$$
E_{\mathrm{d}}(n \Delta t)=\sum_{k=n-N}^{N} E(k \Delta t)
$$

This equation indicates the decaying process.

As an example of the calculation mentioned above, the time transition of the total sound energy in the room shown in Fig. 3(a) was calculated under the condition that the total number of pulses $M$ was 10,356 , the discrete time step $\Delta t$ was $0.05 \mathrm{~s}$ and the sound absorption coefficients of the boundary surfaces $\alpha_{i}$ ( $i=1$ to 6 ) were equally 0.02 . As a result, Fig. 3(b) shows the time transition of the total sound energy $E(n \Delta t)$ under the condition of single pulse radiation. In this case, $E(n \Delta t)$ smoothly decreases with the progress of time and approaches to 0 . Figure 3(c) shows the growing process $E_{\mathrm{g}}(n \Delta t)$ and the decaying process $E_{\mathrm{d}}(n \Delta t)$ under the condition that the sound source radiated energy pulses intermittently for $10 \mathrm{~s}(N=200)$. In this result, it can be seen that the value of $E_{\mathrm{g}}(n \Delta t)$ rapidly increases and approaches a constant value at about 2 seconds after the starting time, and after stopping the pulse radiation, the value decreases smoothly. 


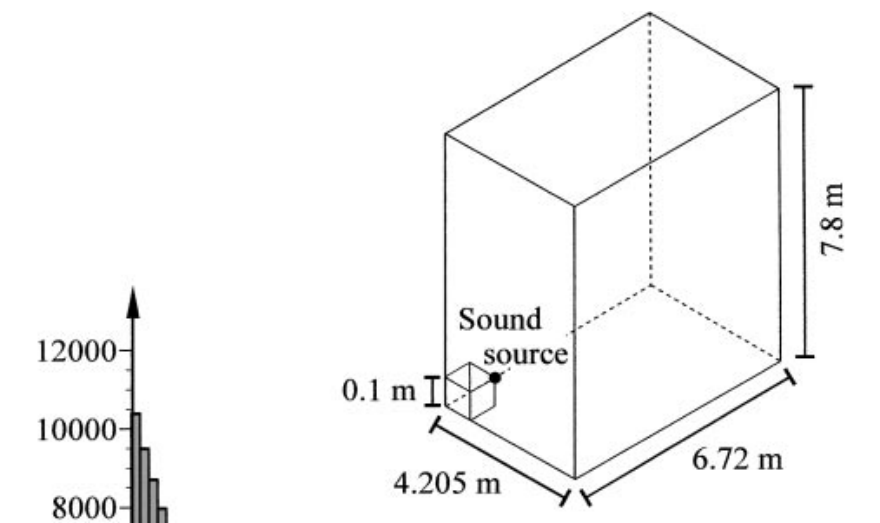

(a) Room under investigation.

area of the room boundaries and $V$ is the volume of the room. When the sound source radiates $M$ energy pulses intermittently at a time interval of $\Delta t$, the equivalent sound power $W$ in calculation can be expressed as follows.

$$
W=\frac{M}{\Delta t}
$$

From Eqs. (6) and (7), the mean sound absorption coefficient of the room boundaries can be expressed as

$$
\bar{\alpha}=\frac{4 V M}{c E_{\mathrm{s}} S \Delta t} .
$$

In the case where a specimen with sound absorption coefficient $\alpha_{\mathrm{m}}$ and area $s$ is mounted on a room boundary and the total acoustic energy in the room is $E_{\mathrm{s}}^{\prime}$, the following relationship exist among $\bar{\alpha}, \alpha_{\mathrm{m}}$ and $E_{\mathrm{s}}^{\prime}$.

$$
\frac{S-s}{S} \bar{\alpha}+\frac{s}{S} \alpha_{\mathrm{m}}=\frac{4 V M}{c E_{\mathrm{s}}^{\prime} S \Delta t}
$$

where the left side indicates the mean sound absorption coefficient of the room boundaries including the specimen. From Eqs. (8) and (9), the sound absorption coefficient of the specimen is given as follows.

$$
\alpha_{\mathrm{m}}=\frac{4 V M}{c s \Delta t}\left\{\frac{1}{E_{\mathrm{s}}^{\prime}}-\left(1-\frac{s}{S}\right) \frac{1}{E_{\mathrm{s}}}\right\}
$$

\section{VARIATION OF THE SOUND ABSORPTION COEFFICIENT DUE TO THE DIFFERENCE OF ROOM SHAPE}

Using the calculation method mentioned above, the sound absorption coefficient of the same specimen set in the reverberation rooms with different shapes was examined. The room shapes under investigation in this study are shown in Fig. 4; a rectangular parallelepiped room (Type 1), a room with irregular shape without any parallel surface (Type 2), a cubic room (Type 3), and the room with the same plan as Type 1 but one wall is slanted (Type 4). As the sound absorption coefficient of the room boundaries, 0.02 was evenly assumed for all of the four rooms. The sound absorption coefficient $\alpha_{\mathrm{m}}$ of the specimen under investigation was assumed in three steps of 0.2 (No. 1), 0.5 (No. 2) and 0.9 (No. 3) and the specimen was located in each room as shown in Fig. 4.

The sound source was located at a corner of each room as shown in Fig. 4, and its sound radiation condition was set as the total number of the energy pulses $M=10,356$ and the time interval $\Delta t=0.05 \mathrm{~s}$. After $10 \mathrm{~s}(N=200)$ from the start of pulse radiation, the acoustic energy density in each room was regarded as steady state.

Figure 5 shows the calculation results. For the specimen No. 1, the calculated values for all of the reverberation rooms are in good agreement with the assumed value 0.2.

where $c$ is the speed of sound, $\bar{\alpha}$ is the mean sound absorption coefficient of the room boundaries, $S$ is the total 


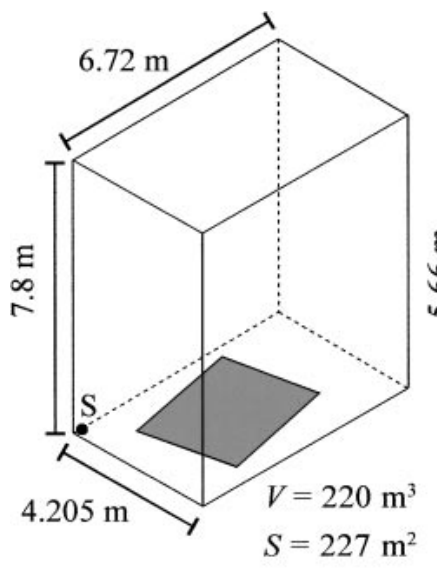

Type 1

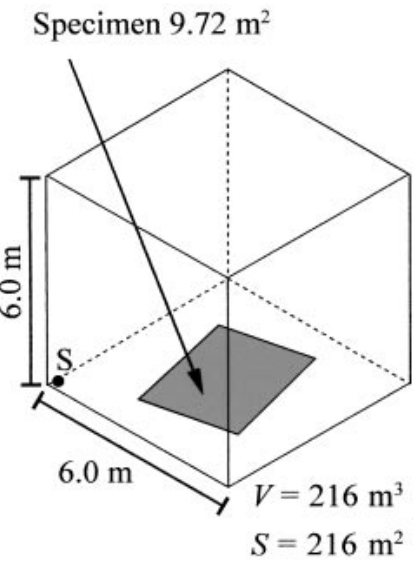

Type 3

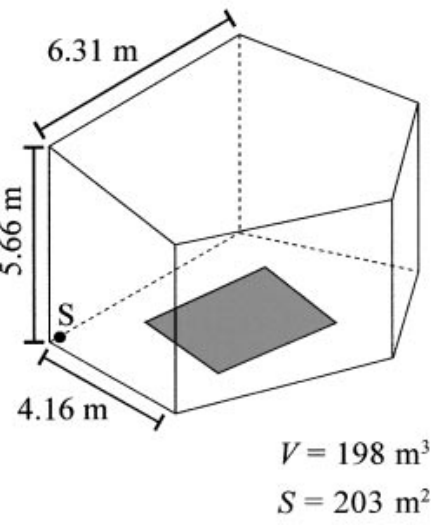

Type 2

Type 4

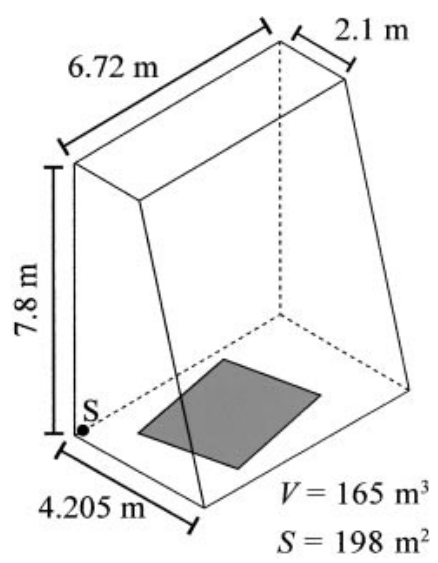

Fig. 4 Room shapes under investigation.

When examining the difference of room shape, the calculated values are in fairly good agreement with the assumed values in the cases of the irregular-shaped rooms Type 2 and Type 4 for all of the specimens. On the other hand, in the cases of the rectangular parallelepiped room Type 1 and the cubic room Type 3 the calculated values are lower than the assumed values for the specimens No. 2 and No. 3. In these cases, the tendency is seen that the discrepancies between the assumed value and the calculated one are larger as the sound absorption coefficient is higher. When comparing the results for the rooms Type 1 and Type 2, it is seen that the calculated values are lower for the rectangular room than those for the irregular room in the cases of the specimens No. 2 and No. 3. This fact might be attributed to the difference of the degree of sound diffusivity in the rooms.

In reverberation rooms, discrete panel diffusers are often installed to make the sound diffusion higher. The effect of this kind of diffusion treatment was investigated for the rooms Type 1 and Type 2. As the diffusers, rectangular panels of $0.9 \mathrm{~m} \times 1.9 \mathrm{~m}$ area were randomly positioned in the rooms and the number of the panels was changed in six steps, $0,1,4,8,16$ and 20. Figure 6 shows
- Specimen No.1 $\alpha_{\mathrm{m}}=0.2$

$\Delta$ Specimen No. $2 \alpha_{\mathrm{m}}=0.5$

- Specimen No.3 $\alpha_{\mathrm{m}}=0.9$

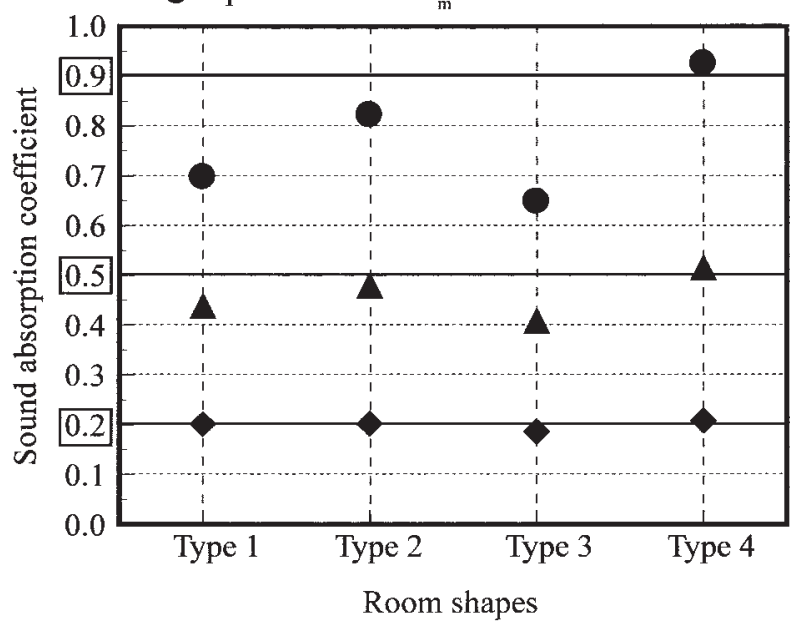

Fig. 5 Calculation results of sound absorption coefficient for the three specimens.

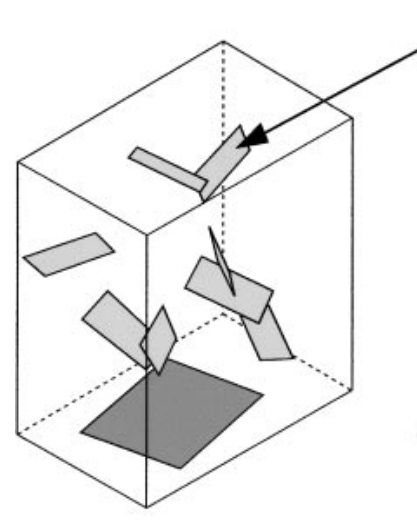

Type 1

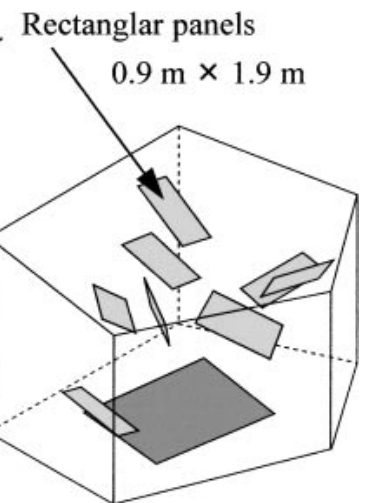

Type 2
Fig. 6 Examples of arrangement of eight panels for the rooms Type 1 and Type 2 .

the case where eight panels are installed in the two rooms.

The calculation results for the case where the specimen No. $3\left(\alpha_{\mathrm{m}}=0.9\right)$ was set and the number of the diffusers was changed are shown in Figs. 7(a) and (b) for the rooms Type 1 and Type 2, respectively. In these results, the calculated value of sound absorption coefficient monotonously increased from 0.70 to 0.91 for the room Type 1 and 0.82 to 0.95 for the room Type 2 , respectively, with the increase of the number of the diffusers.

In order to examine the calculation results mentioned above, the data obtained in real measurement are shown in Fig. 8. In the measurement, two real reverberation rooms with the same shapes and dimensions of the rooms Type 1 and Type 2 were used with and without diffuser panels and the sound absorption coefficient of glass fiber board with $32 \mathrm{~kg} / \mathrm{m}^{3}$ density and $50 \mathrm{~mm}$ thickness was measured by 

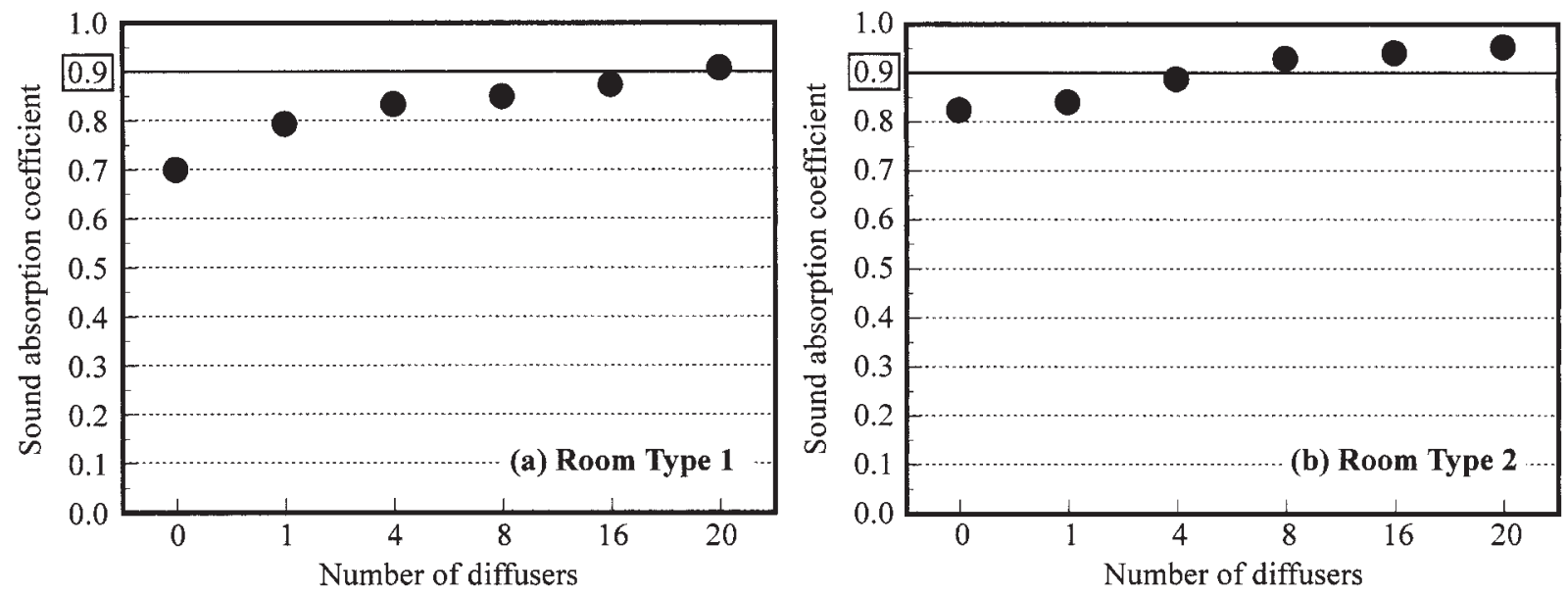

Fig. 7 Calculation results for the cases where the specimen No. $3\left(\alpha_{\mathrm{m}}=0.9\right)$ was set and the number of diffusers was changed.
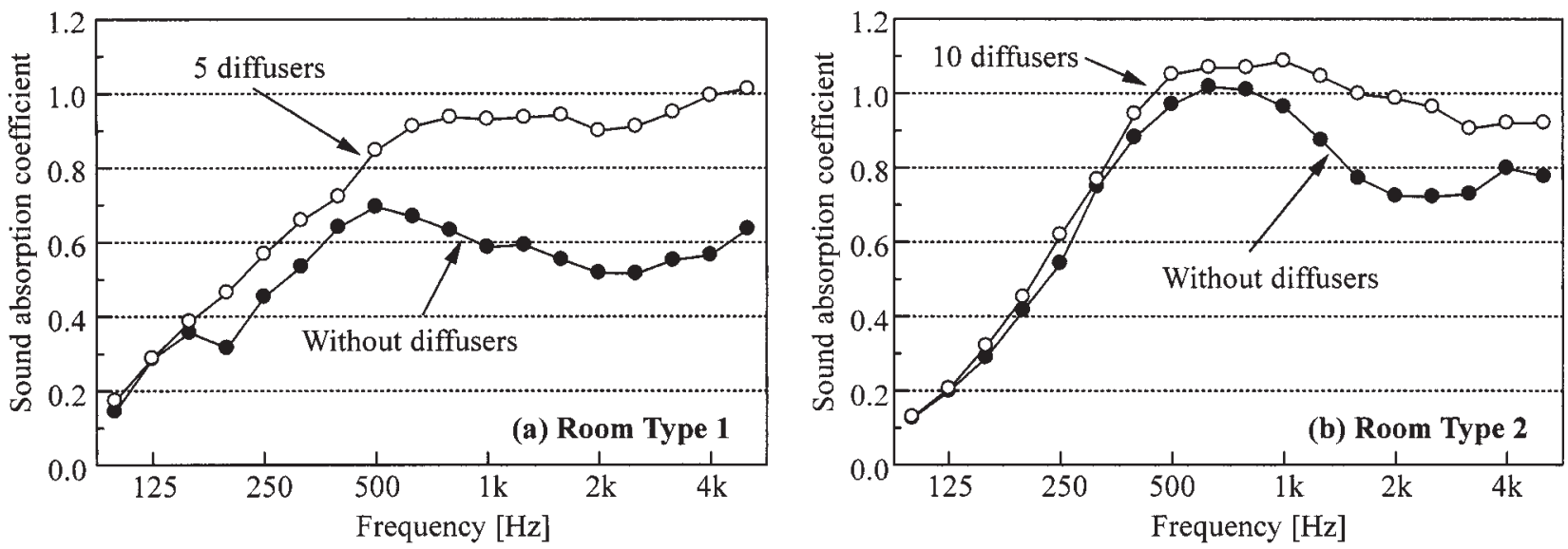

Fig. 8 Measurement results of sound absorption coefficient of glass fiber board with $32 \mathrm{~kg} / \mathrm{m}^{3}$ density and $50 \mathrm{~mm}$ thickness.

the interrupted noise method [7]. In these results, it is also seen that the sound absorption coefficient increases when installing the sound diffusers. Here, it should be noted that the effectiveness of the sound diffusers is higher in the room Type 1 than in the room Type 2 . The same tendency can be seen in the results of the numerical simulation shown in Figs. 7(a) and (b).

\section{DIFFUSIVITY IN THE SOUND FIELD}

As was seen in the results described above, the value of the sound absorption coefficient of a specimen measured by the reverberation room method can be different by the difference of room shape and by the effect of the sound diffusers. This might be attributed to the difference of the degree of sound diffusivity in the room.

The ideal "diffuse sound field" is characterized by the spatial uniformity of acoustic energy density and the isotropy of acoustic energy flow everywhere in the sound field. In order to assess the sound diffusivity in a room, a lot of theoretical and experimental investigations have been conducted [8-12].

By the numerical simulation technique used in this study, the acoustic energy and the incidence angle to the room boundary can be calculated for each sound particle and the isotropy of acoustic energy flow can be assessed indirectly. Accordingly, the sound diffusivity in the room with and without the test specimen was investigated as follows.

\subsection{Calculation Method}

Let us suppose sound power incident to a smooth surface in a diffuse sound field as shown in Fig. 9. In this case, the sound power incident to an infinitesimal area $d S$ with an incidence angle $\theta$ is expressed as follows.

$$
\begin{aligned}
\Delta P(\theta) & =\frac{e_{\mathrm{s}} d S}{4 \pi} \cos \theta \sin \theta \Delta \theta \int_{0}^{2 \pi} d \varphi \int_{0}^{c} d r \\
& =\frac{c e_{\mathrm{S}} d S}{4} \Delta \theta \sin 2 \theta
\end{aligned}
$$

where $e_{\mathrm{S}}$ denotes acoustic energy density and $c$ is the speed 


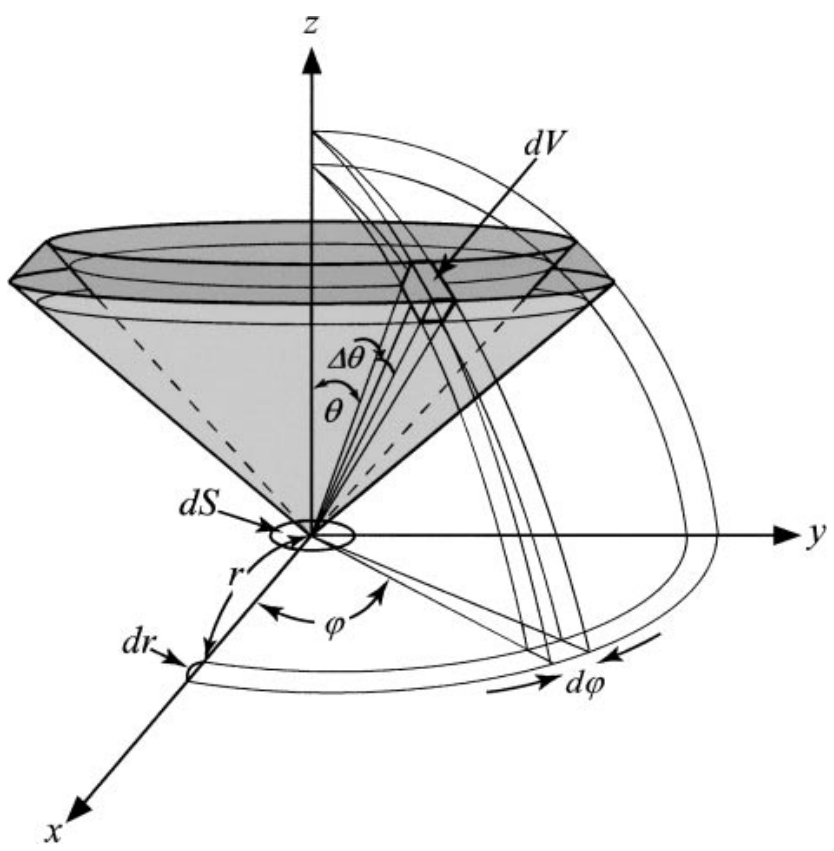

Fig. 9 Sound power incidence to a smooth surface in a diffuse sound field.

of sound. Equation (11) indicates that the sound power incident to a wall is a function of the incidence angle $\theta$ in the diffuse sound field.

In the numerical analysis method applied in this study, the sound power incident to a room boundary can be expressed as a function of the incidence angle by integrating the sound power of the sound ray incident to the room boundary at every time of incidence and by distributing it into every unit angle. In section 2.1., the energy of pulses incident to the wall $w_{i}$ with the incidence angle between $(j-1) \Delta \theta$ and $j \Delta \theta$ during the time interval between $(n-1) \Delta t$ and $n \Delta t$ is denoted as $\Delta E_{i}(j \Delta \theta, n \Delta t)$. Accordingly, the sound power incident to the wall $w_{i}$ under steady state condition with the incidence angle between $(j-1) \Delta \theta$ and $j \Delta \theta$ is expressed as follows.

$$
\Delta P_{\mathrm{s}, i}(j \Delta \theta)=\frac{1}{\Delta t} \sum_{n=0}^{\infty} \Delta E_{i}(j \Delta \theta, n \Delta t)
$$

The sound diffusivity during the decaying process can be assessed by examining the time transition of the incident power. The time transition of the sound power incident to the wall $w_{i}$ with the incidence angle between $(j-1) \Delta \theta$ and $j \Delta \theta$ is expressed as follows.

$$
\Delta P_{\mathrm{d}, i}(j \Delta \theta, n \Delta t)=\frac{1}{\Delta t} \sum_{k=n-N}^{N} \Delta E_{i}(j \Delta \theta, k \Delta t)
$$

\subsection{Sound Diffusivity in Steady State}

In order to examine the sound diffusivity in steady state, the total amount of sound power incident to the room boundaries was calculated. This calculation was performed for the four types of reverberation rooms under the condition of with and without the specimen as a function of a discrete incidence angle $\Delta \theta=\pi / 18$. In the calculation, it was assumed that the sound field becomes steady state after $10 \mathrm{~s}(\Delta t=0.05 \mathrm{~s}$ and $N=200)$ from the start of pulse radiation.

As the reference value to be compared with the calculation results, the angular dependence of total sound power incident to the room boundaries in the ideally diffuse sound field expressed by the following equation was used. This equation can be derived from Eq. (11).

$$
\Delta P(\theta)=\frac{c e_{\mathrm{s}, \mathrm{T}}}{4} S \Delta \theta \sin 2 \theta
$$

where $e_{\mathrm{s}, \mathrm{T}}$ denotes the acoustic energy density in the diffuse sound field, $\theta$ is the incidence angle and $S$ is the total area of the room boundaries. Here, $e_{\mathrm{S}, \mathrm{T}}$ is related to the sound power of the source $W$ and the mean sound absorption coefficient of the room boundaries $\bar{\alpha}$ as follows.

$$
e_{\mathrm{s}, \mathrm{T}}=\frac{4 V W}{c S \bar{\alpha}}
$$

From Eqs. (14) and (15), the angular dependence of sound power incident to the room boundaries can be expressed as follows.

$$
\Delta P(\theta)=\frac{V W}{\bar{\alpha}} \Delta \theta \sin 2 \theta
$$

The calculation results for the four reverberation rooms without and with the specimens No. $1\left(\alpha_{\mathrm{m}}=0.2\right)$ and No. 3 $\left(\alpha_{\mathrm{m}}=0.9\right)$ are shown in Figs. 10 and 11, respectively, as the function of the incidence angle. In these figures, the closed circles indicate the theoretical values of the sound power under the assumption of diffuse sound field. In the results for the case of without the specimen shown in Fig. 10, the calculated sound power and the theoretical value are in good agreement for all of the room shapes. This means that a high sound diffusivity is realized in all of the room shapes under the condition of without the specimen.

On the other hand, when setting the specimen in the rooms, the calculation results are different from the theoretical values as shown in Fig. 11. Especially in the results for the rooms Type 1 and Type 3 with the specimen No. $3\left(\alpha_{\mathrm{m}}=0.9\right)$, the tendency is seen that the pattern is distorted from the theoretical curve and the incident sound power increases at smaller incidence angle. Furthermore, the total amount of sound power of the calculation result is larger than the theoretical values for all types. Such a tendency becomes remarkable as the absorption coefficient becomes high.

Figure 12 shows the calculation results of the angular dependence of the power incident to the surface of the 

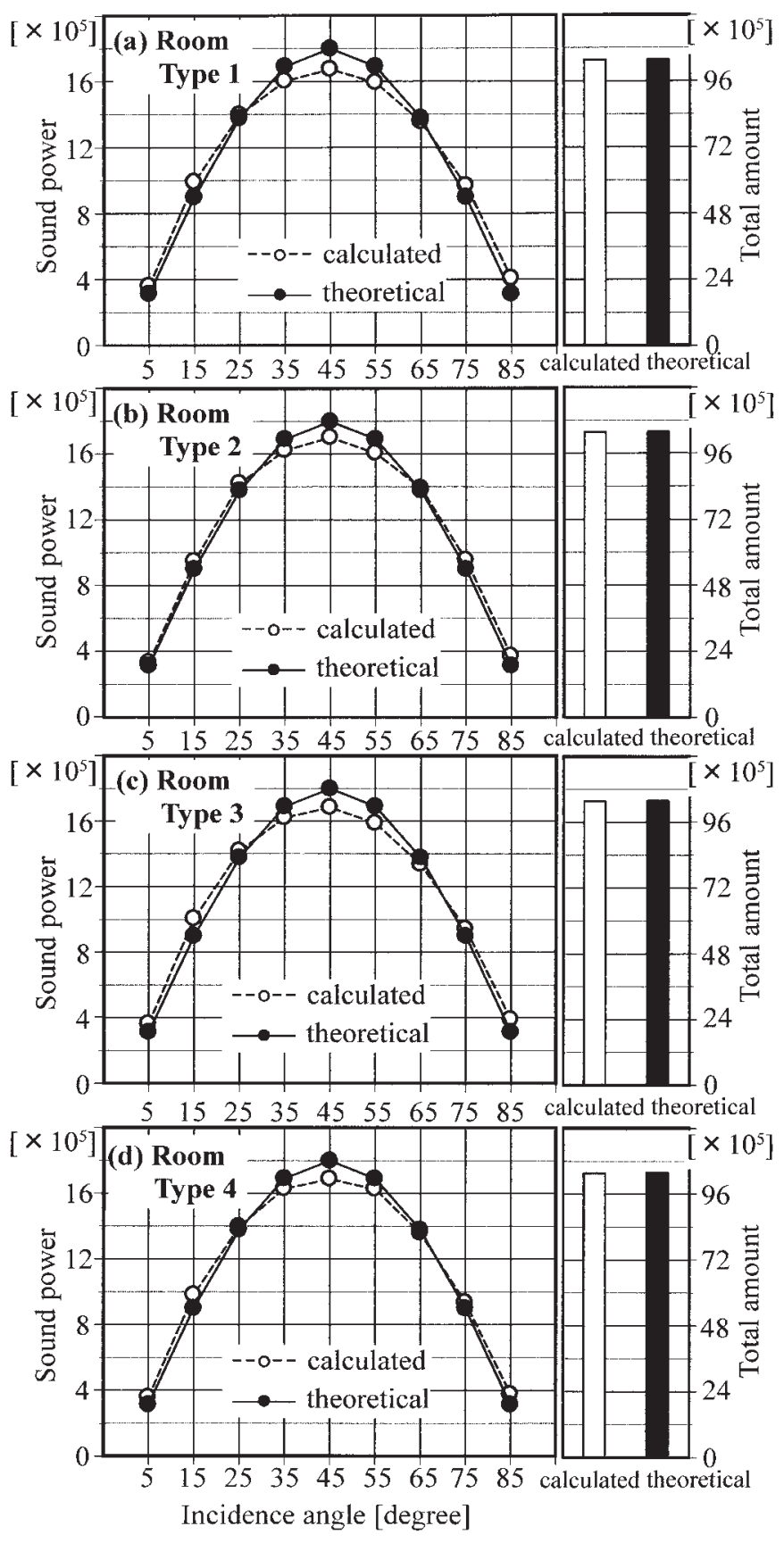

Fig. 10 Calculation results of the angular dependence of the sound power incident to the total area of the room boundaries for the four reverberation rooms without the specimen.

specimen. For all of the rooms, the angular dependence is much different from the theoretical ones obtained under the assumption of diffuse sound field. In the results for the rooms Types 1, 3 and 4, it is seen that the pattern inclines toward the grazing angle (large incidence angle).

In the cases of the rooms Type 1 and Type 3 which consist of three pairs of parallel walls, a contrary tendency is seen between the angular dependence of the power incident to the total area of the room boundaries shown in Fig. 11 and that to the area of the specimen shown in Fig. 12. That is, the pattern of the angular dependence of
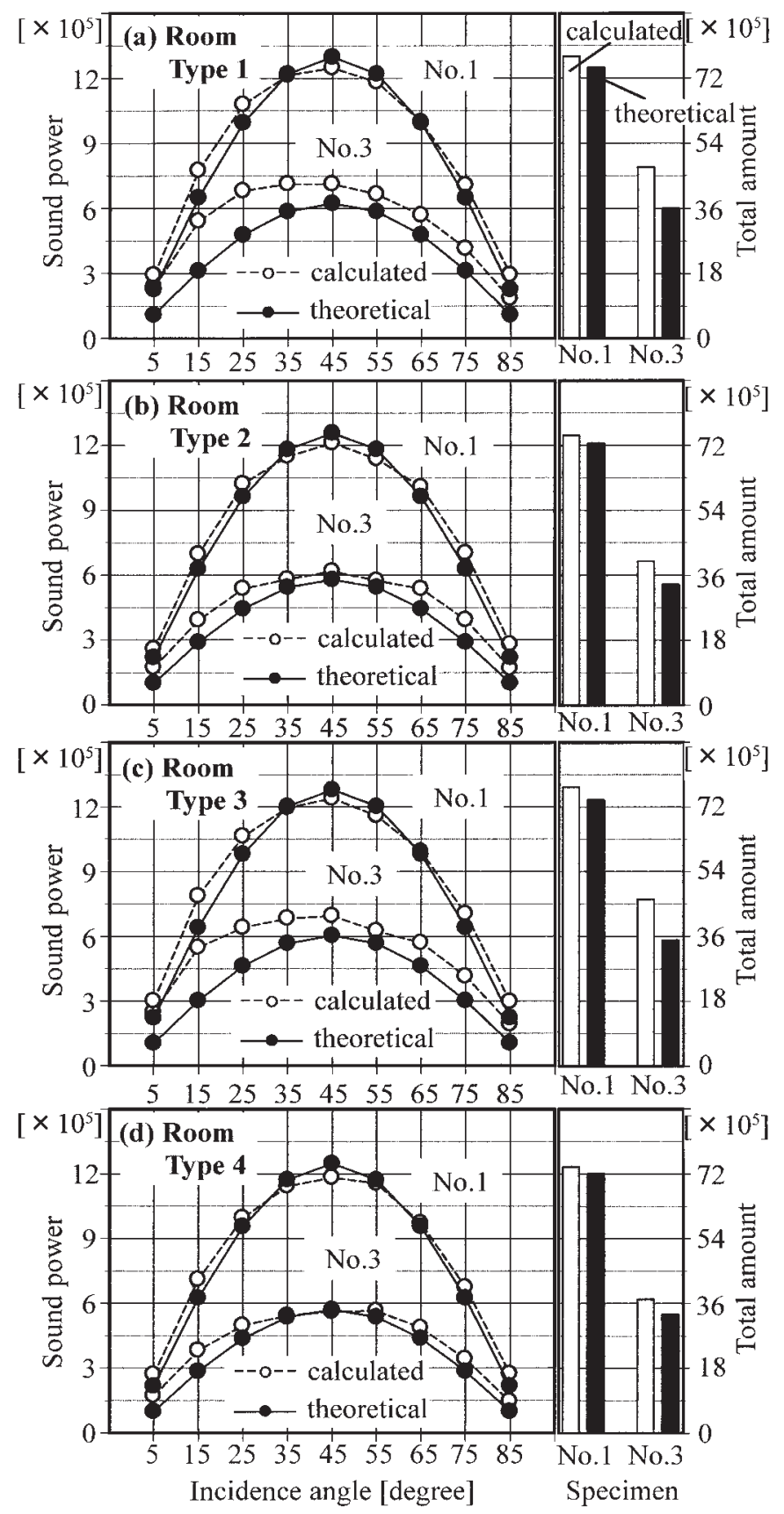

Fig. 11 Calculation results of the angular dependence of the sound power incident to the total area of the room boundaries for the four reverberation rooms with the specimens No. $1\left(\alpha_{\mathrm{m}}=0.2\right)$ and No. $3\left(\alpha_{\mathrm{m}}=0.9\right)$.

the power incident to the total area inclines toward normal incidence (small incidence angle), while that to the surface of the specimen inclines toward grazing angles. This fact can be explained as follows. The sound particles radiated from the sound source make specular reflections on the room boundaries. In the rectangular or cubic reverberation room consisting of parallel surfaces, most of the particles propagate between the parallel surfaces. The acoustic energy of the particles propagating between a pair of parallel surfaces where the specimen is included rapidly decrease due to the sound absorption by the specimen. 

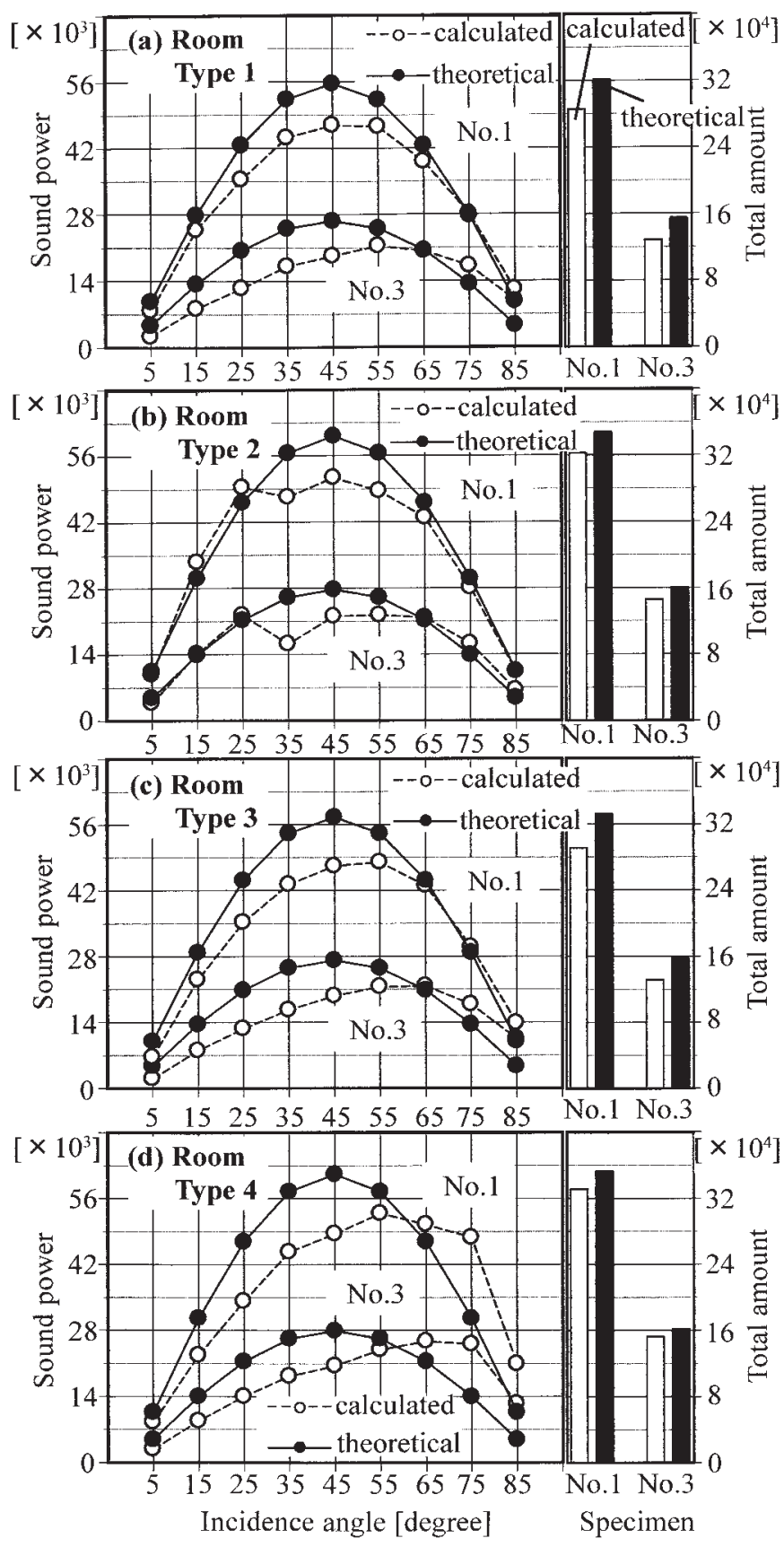

Fig. 12 Calculation results of the angular dependence of the sound power incident to the surface of the specimens No. $1\left(\alpha_{\mathrm{m}}=0.2\right)$ and No. $3\left(\alpha_{\mathrm{m}}=0.9\right)$.

Consequently, the power incident to the surface of the specimen with almost normal direction decreases and the angular dependence of the power incident to the total room surface tends to incline to grazing angle. On the other hand, the acoustic energy of the sound particles propagating between the other pairs of parallel room surfaces remains for a long time. These sound particles hit the room boundaries many times at almost normal incidence angle and consequently the pattern of the angular dependence of the power incident to the total room surface tends to incline toward the normal angle.
Number of diffusers :

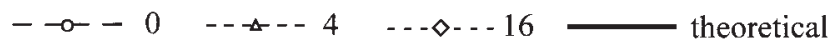

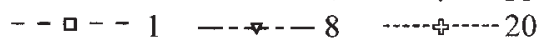
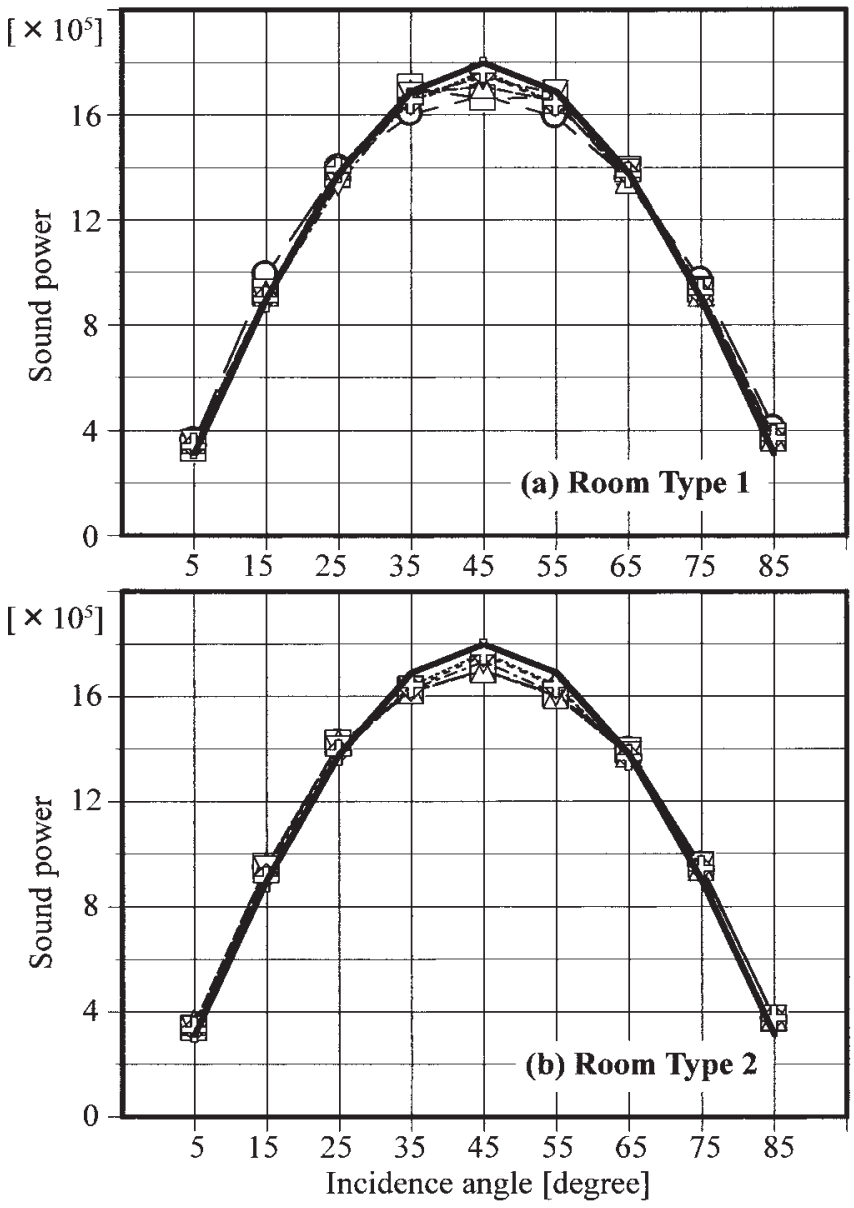

Fig. 13 Variation of the angular dependence of the incident power when changing the number of diffusers for the rooms Type 1 and Type 2 without the specimen.

A further investigation was performed by considering the effect of sound diffusers. Figures 13 and 14 show the variations of the angular dependence of the incident power when increasing the number of the diffusers in the rooms Type 1 and Type 2 . In the case of without the specimen shown in Fig. 13, the calculation results are in good agreement with the theoretical patterns for diffuse sound field in both rooms. It is the same tendency as is seen in Fig. 10 and consequently it can be said that high sound diffusion is realized in both rooms when not including the specimen. On the other hand, Fig. 14 shows the calculation results for the two rooms with the specimen No. 3 $\left(\alpha_{\mathrm{m}}=0.9\right)$. In this result, it is seen that the angular dependence pattern approaches the theoretical values with the increase of the number of diffusers. This fact indicates that the randomly positioned diffusers are effective to make the sound field diffuse even in the case where the specimen is contained. 
Number of diffusers :

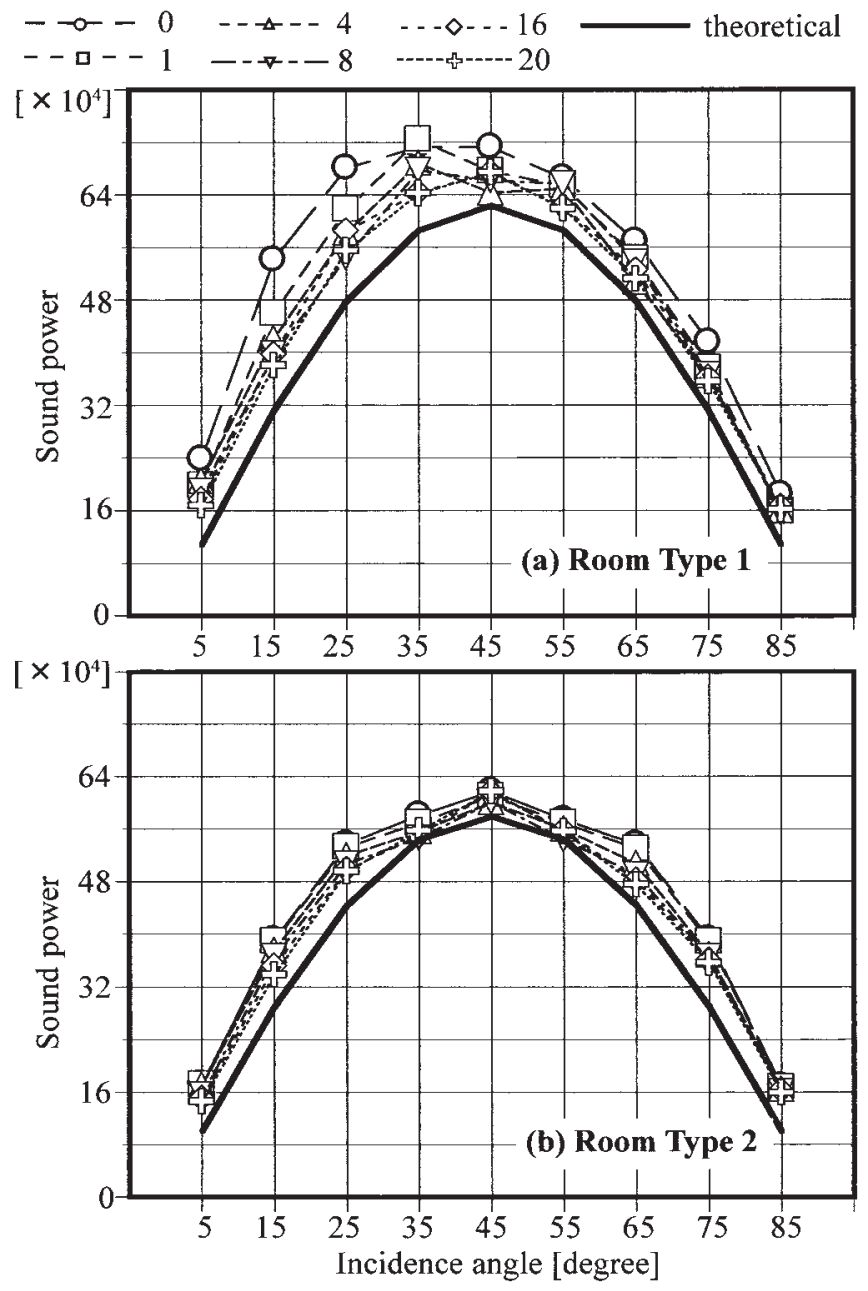

Fig. 14 Variation of the angular dependence of the incident power when changing the number of diffusers for the rooms Type 1 and Type 2 with the specimen No. $3\left(\alpha_{\mathrm{m}}=0.9\right)$.

\subsection{Sound Diffusivity during Transient Process}

In actual measurements of sound absorption coefficient of materials according to ISO 354 and JIS A 1409, reverberation time is measured. Therefore, it is also necessary to evaluate the transient property of the sound field and the time transition of the degree of sound diffusivity during the decaying process in the reverberation rooms was examined.

For all of the reverberation rooms under investigation, the angular dependence of the sound power incident to the total surface of each room and that to the surface of the specimen were calculated for each time period of $0.05 \mathrm{~s}$ during the decaying process.

Figures 15(a-1) to (a-4) show the calculation results of the time transition of the sound power incident to the total room surface under the condition without the specimen. In this case, the pattern of the angular dependence keeps almost symmetrical form which is similar to the pattern calculated for diffuse sound field. On the other hand, Figs. $15(b-1)$ to (b-4) show the results in the case where the specimen No. 3 was set. In these results, it is seen that the patterns are distorted to a greater or lesser extent and especially in the rooms Type 1 and Type 3, the distortion is getting stronger with the passage of time. Figures 15(c-1) to (c-4) show the calculation results of the angular dependence of the acoustic power incident to the specimen. In these results, it is seen that the distortion of the patterns is much stronger as compared to the former results. The tendency that the pattern inclines toward grazing angle with the passage of time is observed for all of the rooms in this case, too.

In order to assess the degree of distortion of the angular dependence, the mean incidence angle defined by the following equation was calculated for each pattern.

$$
\bar{M}(n \Delta t)=\frac{\sum_{j} \Delta P_{\mathrm{d}, i}(j \Delta \theta, n \Delta t) \cdot(j-1 / 2) \Delta \theta}{\sum_{j} \Delta P_{\mathrm{d}, i}(j \Delta \theta, n \Delta t)}
$$

Under the assumption of diffuse sound field, the mean incidence angle is equal to 45 degrees and therefore the difference between $\bar{M}(n \Delta t)$ and 45 degrees can be considered to indicate the degree of sound diffusivity. Figures 16(a) to (c) show the time transition of the mean incidence angles $\bar{M}(n \Delta t)$ obtained from the results shown in Fig. 15. In these results, it is seen that the mean incidence angle leaves from 45 degrees with the passage of time and it means that the degree of sound diffusivity gradually decreases. In Fig. 16(c) showing the $\bar{M}(n \Delta t)$ for the surface of the specimen, it is observed that the temporal variation of the sound diffusivity is different by the difference of the room shape and it can be said that the room Type 2 has the highest sound diffusivity among the four rooms under investigation in this study.

Figure 17 shows the comparison of the temporal variation of $\bar{M}(n \Delta t)$ between the rooms Type 1 and Type 2 when changing the number of the diffusers. In these results, it is seen that the sound diffusivity increases with the increase of the number of the diffusers in both rooms. Especially in the room Type 1, the diffusivity of the sound field can be much improved by introducing the diffusers.

\section{CONCLUSIONS}

The sound absorption coefficient measurement in a reverberation room was investigated by a numerical simulation based on the geometrical acoustics. As a result, it has been confirmed that the sound absorption coefficient of the same specimen can much differ by the differences of room shape and diffusing treatment.

In order to evaluate the degree of sound diffusivity in a room, the angular dependence of the sound power incident 
Room Type 1
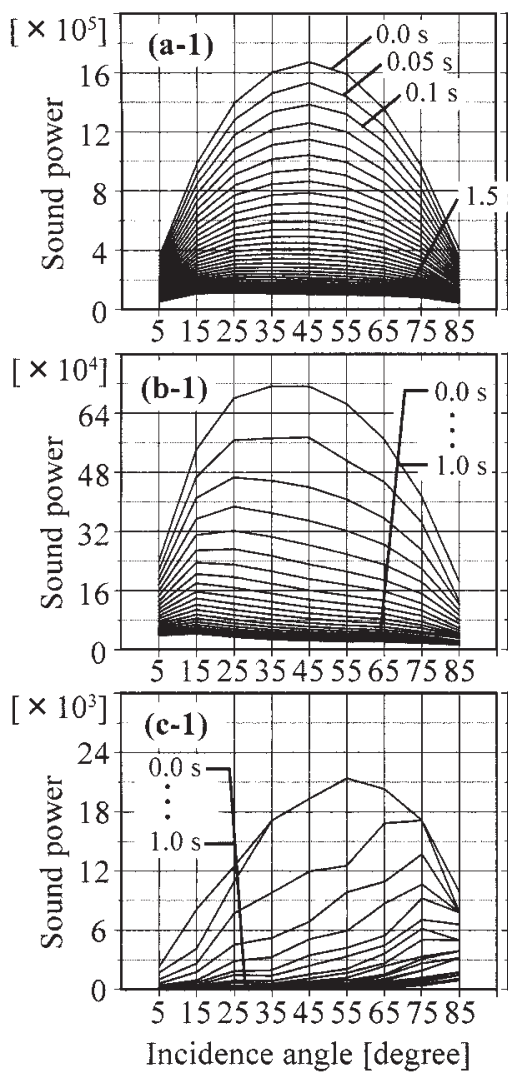

Room Type 2

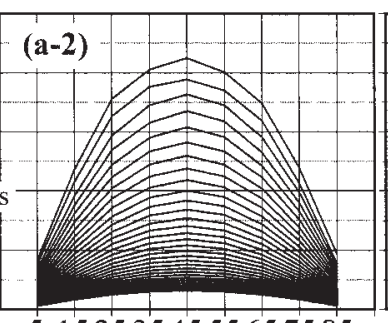

51525354555657585

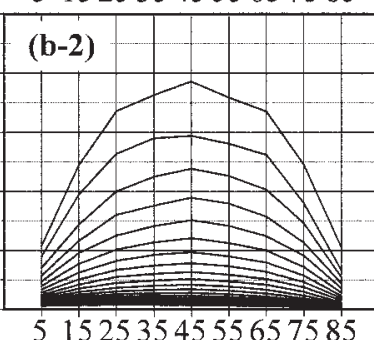

(c-2)

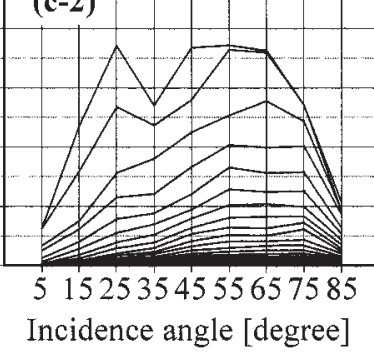

Room Type 3

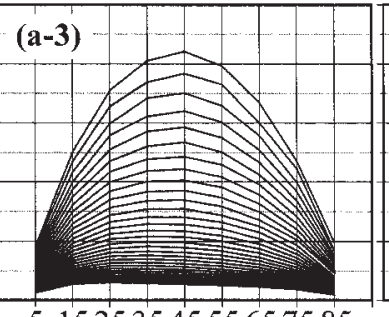

51525354555657585

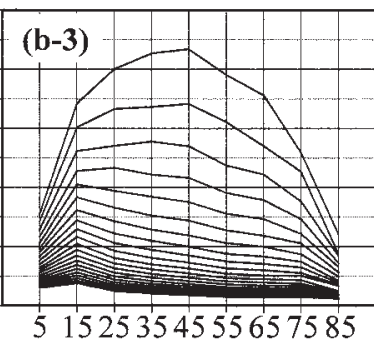

(c-3)

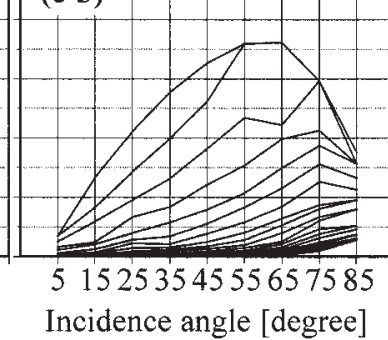

Room Type 4

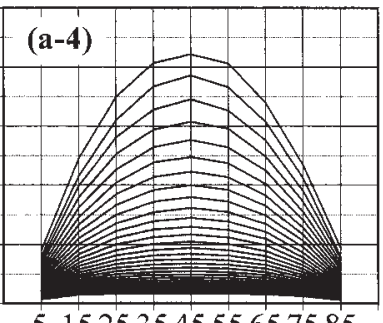

51525354555657585

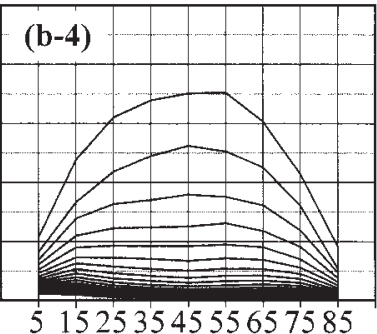

(c-4)

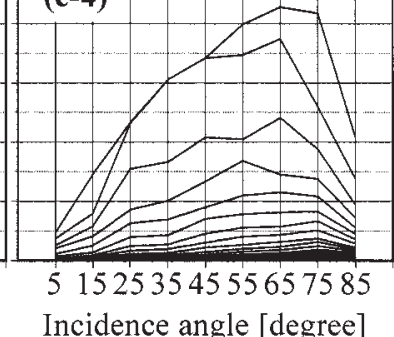

Fig. 15 Calculation results of the angular dependence of the sound power during decaying process.

(a-1) to (a-4) Time transition of the sound power incident to the total room surface under the condition without the specimen.

(b-1) to (b-4) Time transition of the sound power incident to the total room surface under the condition with the specimen

No. $3\left(\alpha_{\mathrm{m}}=0.9\right)$.

(c-1) to (c-4) Time transition of the sound power incident to the surface of the specimen No. $3\left(\alpha_{\mathrm{m}}=0.9\right)$.
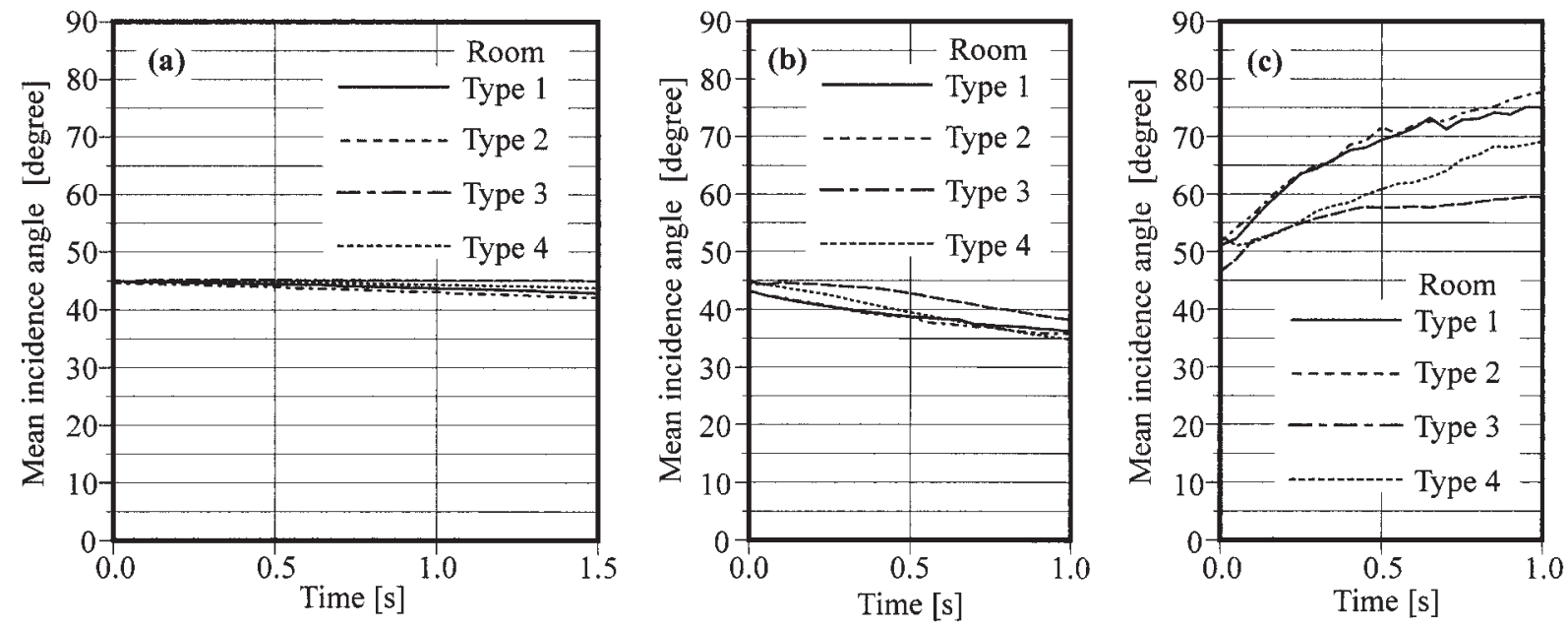

Fig. 16 Time transition of the mean incidence angle $\bar{M}(n \Delta t)$.

(a) $\bar{M}(n \Delta t)$ for the total room surface under the condition without the specimen.

(b) $\bar{M}(n \Delta t)$ for the total room surface under the condition with the specimen No. $3\left(\alpha_{\mathrm{m}}=0.9\right)$.

(c) $\bar{M}(n \Delta t)$ for the surface of the specimen No. $3\left(\alpha_{\mathrm{m}}=0.9\right)$. 


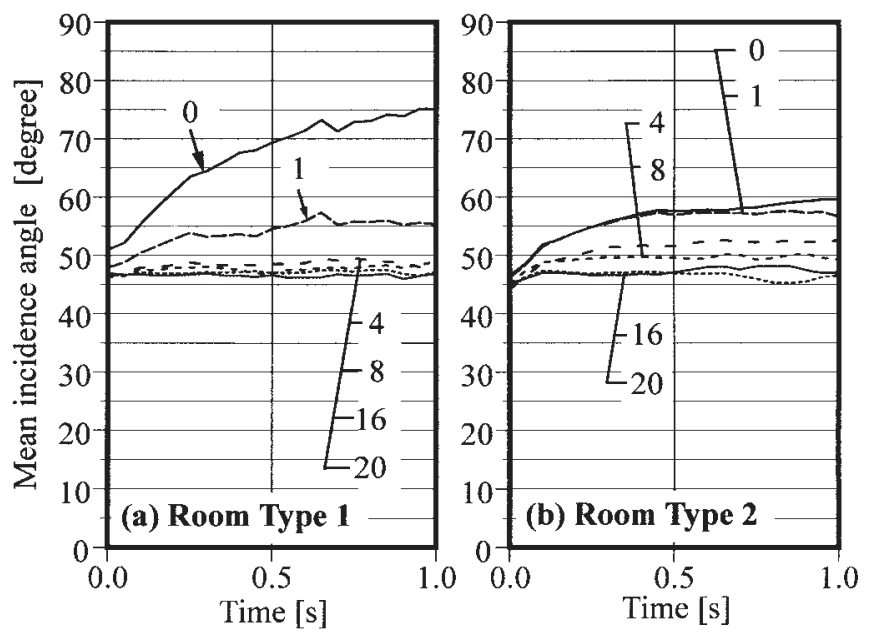

Fig. 17 Comparison of the temporal variation of $\bar{M}(n \Delta t)$ between the rooms Type 1 and Type 2 when changing the number of diffusers. The integer indicates the number of diffusers.

to the room boundary and to the surface of the specimen were examined by the numerical simulation. As a result, the followings have been found.

1) The sound diffusivity in a reverberation room much decreases when sound absorbing material with high absorption property is locally set.

2) Under such a condition, the sound diffusivity during the reverberation decay process decreases with the progress of time.

3) The installation of sound diffusers is very effective to increase the sound diffusivity in a room. This effect is remarkable especially for rectangular parallelepiped reverberation rooms.

In this study, the calculation has been performed based on the geometrical acoustics and the wave nature of sound can not be treated. Therefore there must be a limit to the findings obtained here. For example, the edge effect caused by the wave phenomenon can not be treated in this study. The sound diffusivity dependent upon both of the room volume and frequency of the sound also can not be discussed. However, the discrepancies of the measurement results of sound absorption coefficient due to the differences of reverberation room shape and sound diffusing treatment can be explained to some extent by this simulation analysis. Further, this kind of sound field analysis would be effective for the design of reverberation rooms and the arrangement of sound diffusers.

\section{REFERENCES}

[1] C. W. Kosten, "International comparison measurements in the reverberation room," Acustica, 10, 400-411 (1960).

[2] Y. Makita, M. Koyasu, M. Nagata and S. Kimura, "Investigations into the precision of measurement of sound absorption coefficients in a reverberation room (I) - The 3rd round robin test and the investigations on the diffusitivity of sound field-," J. Acoust. Soc. Jpn. (J), 24, 381-392 (1968).

[3] Y. Makita, M. Koyasu, M. Nagata and S. Kimura, "Investigations into the precision of measurement of sound absorption coefficients in a reverberation room (II) - Experimental studies on the method of measurement of the reverberation time and the 4th round robin test-," J. Acoust. Soc. Jpn. (J), 24, 393-402 (1968).

[4] K. Sato and M. Koyasu, "On the new reverberation chamber with nonparallel walls (Studies on the measurement of absorption coefficient by the reverberation chamber method, II)," J. Phys. Soc. Jpn., 14, 670-677 (1959).

[5] ISO 354:2003, Acoustics-Measurement of sound absorption in a reverberation room.

[6] JIS A 1409:1998, Method for measurement of sound absorption coefficients in a reverberation room.

[7] E. Toyoda, S. Sakamoto and H. Tachibana, "Effect of reverberation room shape on measurement result of sound absorption coefficient — part 1. Experimental study_,"Summaries of Technical Papers of Annual Meeting, AIJ, pp. 225226 (2000).

[8] C. G. Balachandran and D. W. Robinson, "Diffusion of the decaying sound field," Acustica, 19, 245-257 (1967/68).

[9] M. Koyasu and M. Yamashita, "Evaluation of the degree of diffuseness in reverberation chambers by spatial correlation techniques," J. Acoust. Soc. Jpn. (J), 26, 132-143 (1971).

[10] T. J. Schultz, "Diffusion in reverberation rooms," J. Sound Vib., 16, 17-28 (1971).

[11] L. J. Zeng, "The sound distribution in a rectangular reverberation chamber," J. Acoust. Soc. Am., 92, 600-603 (1992).

[12] H. Nelisse and J. Nicolas, "Characterization of a diffuse field in a reverberant room," J. Acoust. Soc. Am., 101, 3517-3524 (1997). 


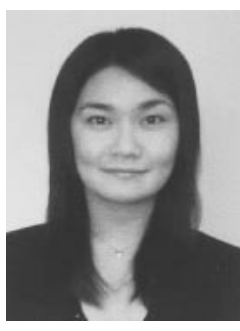

Emi Toyoda was born on September 16, 1977 in Chiba, Japan. She graduated from Toho University in 2000 and received a Master of Science degree from Toho University in 2002. She is currently a researcher at Kobayasi Institute of Physical Research. Her current research interests are the measurement of acoustical properties and numerical analysis of sound fields. She is a member of ASJ, Institute of Noise Control Engineering of Japan and Architectural Institute of Japan.

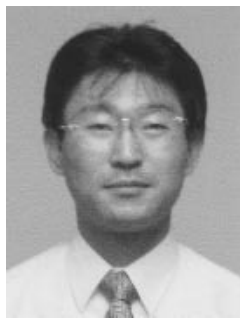

Shinichi Sakamoto was borne on June 10, 1968 in Saitama, Japan. He received B.E., M.E. and Doctor of Engineering degrees from the University of Tokyo in 1991, 1993 and 1996, respectively. Associate Professor of the Applied Acoustic Engineering Laboratory, Institute of Industrial Science, University of Tokyo. His current research interests are in areas of architectural acoustics and environmental noise control engineering. A member of ASJ, INCE/J and AIJ

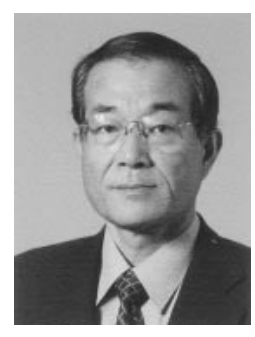

Hideki Tachibana was borne on August 23, 1943 in Tokyo, Japan. Graduated from Faculty of Engineering, Tokyo University in 1967, and received Engineering Doctor from Tokyo University in 1973. Professor of the Applied Acoustic Engineering Laboratory, Institute of Industrial Science, Tokyo University. Board Member, Acoustical Society of Japan (ASJ), 1989-1993, 1995-; President, ASJ, 1995-1997; Chief in Editor, ASJ, 1999-2003; Chair, Technical Committee of Road Traffic Noise, ASJ, 1990-; Board Member, Institute of Noise Control Engineering of Japan (INCE-J), 1990-1996, 2000-; President, INCE-J, 2002-; President, International Institute of Noise Control Engineering (I-INCE), 2004-; Fellow, Acoustical Society of America (ASA), 1996-; Prize of the Architectural Institute of Japan, 1990; Prize of ASJ, 1991; Rayleigh Medal (Prize of IOA), 2002. 